\title{
Arzthaftung
}

\section{Schweigen ist Gold}

\begin{abstract}
Ärztliche Haftpflichtfälle werden in der Regel zivilrechtlich abgewickelt und enden idealerweise mit einem außergerichtlichen Vergleich. Unerfahrene Anwälte leiten manchmal ein Strafverfahren ein, damit der Patient Einsicht in die Krankenakten erhält. In einer solchen Situation sollten Arzt und Praxispersonal ohne anwaltlichen Beistand keinerlei Aussagen machen, mahnte Rechtsanwalt Roland Wehn auf einem Symposium „Arzthaftung - Ihr juristischer Notfallkoffer" im Rahmen des Internistenkongresses.
\end{abstract}

— Ärztliche Haftpflichtfälle sind meist eine zivilrechtliche Angelegenheit. In der Regel geht es um die Durchsetzung einer Schadenersatzforderung wie etwa Verdienstausfall, Schmerzensgeld, Unterhalt für die Angehörigen etc. In den meisten Fällen kommt es - sollte das Verfahren nicht abgelehnt werden - zu einer außergerichtlichen Einigung, die für alle Beteiligten (Versicherer, Patient, Arzt) erstrebenswert ist.

\section{Besser außergerichtlich einigen, als} Opfer der Lokalpresse zu werden Denn wenn es doch zu einem Prozess kommt, hat der Arzt oft auch die örtliche Presse auf dem Hals. Selbst wenn die Unschuld des Arztes später bewiesen wird, bleiben Patienten weg. Umsatzeinbußen von 30-40\% sind dann keine Seltenheit, weiß Jurist Wehn von der DBV-Winthertur.

\section{Strafrechtliche Verurteilungen in einem Prozent aller Haftpflichtfälle}

$\mathrm{Zu}$ einem strafrechtlichen Verfahren kommt es eher selten. Von 4280 Arzthaftpflicht-Schäden, die der DBV-Win- terthur in 2003 gemeldet wurden, wurde in 170 Fällen ein strafrechtliches Ermittlungsverfahren eingeleitet. Bei 43 Ärzten kam es zu Verurteilungen.

Dass Ärzte in Strafrechtsverfahren verurteilt werden, ist deshalb relativ selten, weil bei den Ermittlungen bewiesen werden muss, dass bei dem Patienten sicher ein nachweisbarer Behandlungsfehler vorliegt. So eindeutig ist das eher selten der Fall. Die Gutachten fallen in strafrechtlichen Ermittlungsverfahren deshalb häufig positiv für den Arzt aus. Im Zweifel für den Angeklagten, lautet auch hier der Grundsatz.

\section{Verhaltensregeln für die Praxis im Vermittlungsverfahren}

Dennoch sollte das Thema „Ermittlungsverfahren“ in Praxisbesprechungen angesprochen werden, rät Jurist Wehn. Denn gerne vernimmt die Polizei z.B. Kollegen oder das Praxispersonal als Zeugen. In der Annahme, nicht an der Sache beteiligt zu sein, reden sich nicht nur der verdächtige Arzt, sondern auch die vermeintlichen Zeugen um Kopf und Kragen.

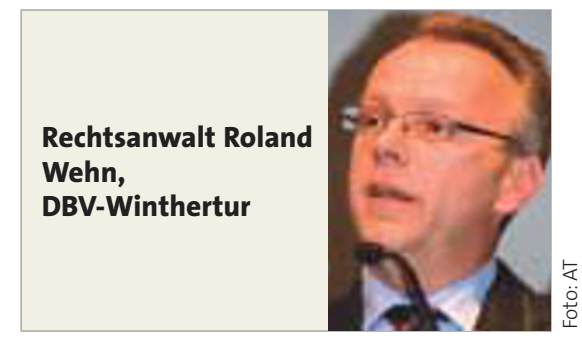

Im Laufe der Ermittlungen kann sich nämlich später herausstellen, dass der Zeuge selbst auch verdächtig ist. Deshalb sollten sowohl der Arzt als auch weitere Kollegen und das Personal grundsätzlich von ihrem Aussageverweigerungsrecht Gebrauch machen. Hier hilft es, z.B. zu sagen: „Ich bin total fertig. Ich kann in dieser Situation keine Aussage machen."

Weiterhin rät Rechtsanwalt Wehn Ärzten, sich in jedem Fall einen auf Arzthaftungsrecht spezialisierten Fachanwalt an die Seite zu holen.

Wann liegt ein Behandlungsfehler vor? Ärzte müssen immer dann haften, wenn ein Behandlungsfehler vorliegt. Aber wann ist das der Fall? „In einer Kurzformel könnte man sagen“, so Rechtsanwalt Wehn, „ein Behandlungsfehler ist jedes Abweichen des Ist-Standards der Behandlung vom zu fordernden Soll-Standard." Und der Soll-Standard orientiert sich dabei an einem „situationsbezogenen Facharztstandard zu jeder Zeit", was natürlich etwas schwammig ist. 
Auch Hausärzte müssen sog. Facharztstandards einhalten

Gutachter prüfen in Haftungsfällen in der Regel, ob der jeweils maßgebliche Facharztstandard eingehalten wurde. Hier sind insbesondere Hausärzte eine gefährdete Gruppe, warnt Rechtsanwalt Wehn. Denn Hausärzte (auf dem Land), die z.B. auch viele Kinder behandeln, müssen sich am Facharztstandard Pädiatrie orientieren. Und ob diese Kenntnisse immer auf dem jeweils aktuellsten Stand sind, dürfte fraglich sein.

Ähnlich gefährdet arbeiten Ärzte im fachübergreifenden Bereitschaftsdienst. Dazu ein Beispiel: Eine Patientin klagte samstags um 22.00 Uhr bei dem
Dienst habenden Arzt (angehender Facharzt Chirurgie) über linksseitige Schmerzen in der Brust. In der Anamnese wurde aufgenommen, dass die Patientin die Pille einnimmt, Raucherin ist und erst vor Kurzem aus Thailand zurückgekehrt war. Der Chirurg entschied sich für eine Röntgenaufnahme der Lunge und befundete eine linksseitige Lungenentzündung. Um 4.35 Uhr wurde der Notarzt zur Patientin gerufen, der den Tod nach Embolie feststellte.

Der Fall lehrt: Der angehende Chirurg hätte den Dienst habenden Internisten hinzuziehen müssen. Da er selbst befundete, hatte der Arzt den fachärztlichen Standard verlassen.

\section{Grobe Behandlungsfehler}

Der schlimmste mögliche Fall in der Arzthaftung ist der Vorwurf eines groben Behandlungsfehlers. Dieser sollte einem Arzt unter gar keinen Umständen unterlaufen, so Rechtsanwalt Wehn. Handelt ein Arzt nämlich grob fahrlässig, dreht sich die Beweislast um: Der betroffene Arzt muss dann beweisen, dass der Schaden auch eingetreten wäre, wenn der Fehler nicht passiert wäre. Ein Beweis, der Ärzten in den seltensten Fällen gelingt.

ANKE THOMAS :

- Quelle: Symposium „Arzthaftung - Ihr Juristischer Notfallkoffer", 113. Kongress der Deutschen Gesellschaft für Innere Medizin, Wiesbaden, 14.-18. April 2007

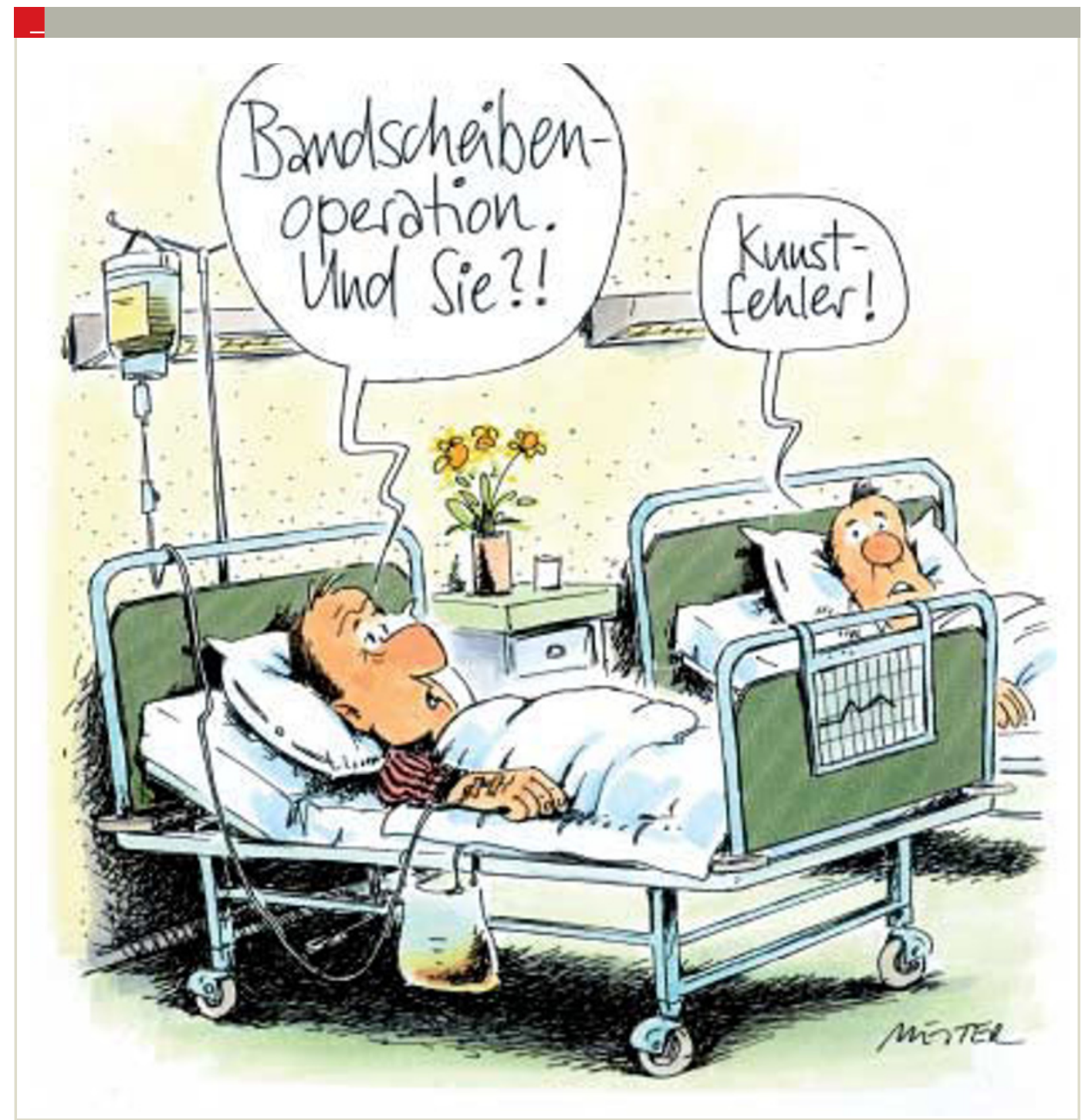

